\title{
Preliminary design of aeroelastically tailored wing box structures with bend-twist coupling
}

\author{
Mihai Mihaila-Andres ${ }^{1, *}$, Paul-Virgil Rosu ${ }^{1}$, Ciprian Larco ${ }^{1}$, Maria Demsa $^{1}$,Lucian Constantin ${ }^{1}$ and Radu Pahonie ${ }^{1}$ \\ ${ }^{1}$ Military Technical Academy "Ferdinand I", Aircraft Integrated Systems and Mechanics Department, 39-49 George Cosbuc, \\ Bucharest, Romania
}

\begin{abstract}
Nowadays the composite materials have become the materials of choice to be used in the new aerospace structures that need to be not only larger and larger in size but also to be better performing in terms of aeroelastic responses inherent to thin-walled, slender structures. The advantage of composite materials airframes stems from their low structural weight which determines lower fuel consumption while preserving at the same time the airworthiness of the designed aircraft. But more important than the fuel consumption, the composite materials allow for the optimal tailoring of its layers in terms of specific design objectives. The paper presents such an aeroelastically tailored load carrying wing model which can passively control specific aeroelastic effects. The article focuses on the bend-twist coupling of the structural response to aerodynamic forces and on the parameter estimation/model updating techniques used to characterize the finite element model of the composite wing. Results are compared and validated with analytical, numerical and experimental data available in published literature.
\end{abstract}

\section{Introduction}

Over the last years there have been extensive research and development activity in the aircraft industry to support the need for larger, more environmentally friendly airframes with low-priced life-cycle costs. Compared to aluminum alloys used in aviation, the composite materials have the clear advantage of excellent strength/weight and stiffness/weight ratios, precise mass distribution, flexibility and durability, better response to shocks, reduced corrosion/fatigue effects and lower maintenance costs $[1,2]$. In addition to these advantages, the designer's capacity of tailoring the stiffness of airframe components made of composite materials is a key element in their recent years dominance [3]. Using the directional stiffness of the plies, the resulting laminated parts can be optimized to build lightweight airframes at the same time circumventing aeroelastic instabilities inherent to the slender, highly flexible airframe components like wings, control surfaces or fuselages [4, 5, 6, 7, 8, 9, 10]. Divergence, flutter, induced drag, lift/control effectiveness and maneuver loads are all aeroelastic instabilities that can be controlled through "the embodiment of directional stiffness into an aircraft structural design to control aeroelastic deformation, static or dynamic, in such a fashion as to affect the aerodynamic and structural performance of that aircraft in a beneficial way" [11]. It is the definition of aeroelastic tailoring given by M. Shirk, T.J. Hertz and T.A. Weisshaar.
According to this definition, the aeroelastic tailoring technique can be seen as a passive method [12] of controlling the aeroelastic response of an airframe structural design within its flight envelope in the absence of any actuator or complementing some other active control method.

The anisotropy of the composite materials in general and the anisotropy of laminated composites in particular is the key of such a control mechanism which can regulate the degree of coupling between the deformations and/or curvatures of the laminated composite structure. The optimized choice of ply stacking sequence can induce a global or even local coupling between extension, bend, twist and shear, for example bend-twist coupling which is important in wing structures or extension-twist coupling which is important in rotor blade structures [13].

It is exactly this bend-twist coupling mechanism that is used in this study to estimate the parameters of the optimum ply stacking lay-up when combined with loads that are causing aeroelastic effects.

An in depth analysis of the optimization process or the aeroelastic tailoring process as defined beforehand shows that we are dealing with a multidisciplinary problem which has multiple objectives and constraints pertaining to disciplines like aerodynamics, structural dynamics or manufacturing engineering.

The multidisciplinary nature of the subject and the need to trade-off the objectives related to dissimilar disciplines was a draw back for the development of aeroelastic tailoring techniques. Things changed in the recent years with the advent of optimization

\footnotetext{
Corresponding author: mihai.mihaila@mta.ro
} 
mathematical tools that evolved from analytical and experimental models to more intricate finite element models. The challenge with these complex finite element optimization models is that meaningful optimum designs can be obtained only if using accurate and efficient numerical methods. Usually accuracy is sacrificed for efficiency when time is a management problem and this is the case we are dealing with since the design phases would require running over and over again the complex finite element models for solving the fluid problem, the structural problem and the optimization problem. In the context of a very competitive aircraft industry, we are confronted then with the second trade-off the designer needs to make when choosing the optimization model. Should it be reliable or fast? The answer should be: fast optimization algorithms to be used in the conceptual design phase and accurate optimization algorithms in the final design phase. [14]

\section{Box-beam analytical model}

In view of the aforementioned introduction, a new fast preliminary design tool for the aeroelastic tailoring of wing structures has been developed that uses a reduced fidelity analytical model in the form of a box-beam loadcarrying structure which has the advantage of faster computational times. The model was used to understand the cause-effect relationship between the lay-up of the laminates and the aeroelastic response of the equivalent wing made of these laminates with focus on the identification of conceptual composite wing structures capable of coupling the bending deformations with the twist deformations.

In the proposed model the wing of an aircraft can be represented as a thin-walled, closed section, box-beam clamped at one end. Using the classical lamination theory, the stresses in the walls of the box beam can be represented by analytical expressions of laminated panels characterized by distinct lay-ups of the plies for upper surface, lower surface and spars.

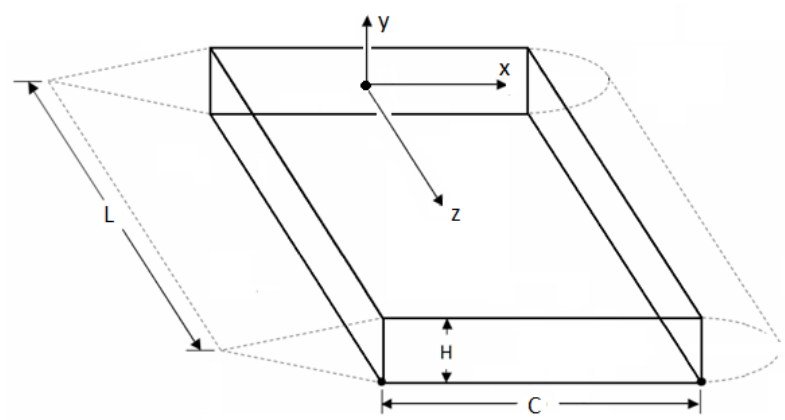

Fig. 1. Generic wing, notations and coordinate system

Considering the invariable cross-section of the box-beam loaded at the free end with generalized forces, one can apply Castigliano's theorem to the strain energy of an elementary beam length to assemble the generalized displacements into an explicit form of cross-sectional constitutive law $[15,16]$. This explicit form can be further simplified if we keep only two generalized forces which are predominant in the aerodynamic loads of a wing (bending moment about the root chord and torque about the span) and neglect the other four $[17,18]$. The new cross-sectional constitutive law can be written then as:

$$
\left\{\begin{array}{l}
H_{x} \\
T_{z}
\end{array}\right\}=\left[\begin{array}{ll}
c_{11} & c_{16} \\
c_{16} & c_{66}
\end{array}\right]\left\{\begin{array}{l}
\theta_{x}^{\prime} \\
\theta_{z}^{\prime}
\end{array}\right\}
$$

where $H_{x}$ is the bending moment, $T_{z}$ is the twist moment, $\theta_{x}, \theta_{z}$ are the torsional rotations and $c_{i j}$ are the bending, twist and bend-twist coupling stiffness coefficients.

The twist and the deflection along the axial direction of the box beam model can be obtained through simple integral operations of the twist rate $\theta_{\mathrm{z}}^{\prime}$ and the bending curvature $\theta_{\mathrm{x}}^{\prime}$ derived from (1):

$$
\begin{gathered}
\theta_{z}^{\prime}=\frac{-c_{16} H_{x}+c_{11} T_{z}}{c_{11} c_{66}\left(1-\beta^{2}\right)} \\
\theta_{x}^{\prime}=\frac{c_{66} H_{x}-c_{16} T_{z}}{c_{11} c_{66}\left(1-\beta^{2}\right)}
\end{gathered}
$$

where

$$
\beta^{2}=\frac{c_{16}^{2}}{c_{11} c_{66}}
$$

Rehfield and Cheung [19] showed that $\beta$ can measure the degree of coupling between the bending and the twist of the wing under aerodynamic loads.

Based on this mathematical description of an idealized wing, the preliminary design tool determines the bend and the twist using as input data the lay-up of the wing surfaces and simple aerodynamic loads. Detailed information on the bend-twist coupling analysis using this tool is presented in [20], where various box-beam cases have been modeled and validated with experimental and numerical data available in the published literature.

\section{Aeroelastically tailored wing box fabrication}

Once validated, the preliminary design tool was used to design a simple composite wing that would exhibit a controllable bend-twist effect. In such a configuration, depending on the desired effect, the lay-up of the upper and lower surfaces would induce a nose-up or nosedown behavior as the multiple test-cases simulated with the preliminary design tools showed. As a consequence, lift efficiency, induced drag, flutter or divergence can be passively controlled by thoughtfully adjusting the lay-up sequence of the wing upper surface and lower surface.

The lay-up sequence of the fabricated wing was decided on the basis of the coupling behavior predicted by the preliminary design tool (Table 1) and the dimensions were restricted by fabrication constraints and by the experimental setup.

All four parts of the box-beam have been fabricated using a vacuum assisted resin transfer method (VARTM) which has the advantage of not requiring an autoclave for the curing process. The unidirectional fabric C100U 
used to fabricate the panels had a nominal mass/area of $100 \mathrm{~g} / \mathrm{m}^{2}$ and a thickness of $0.11 \mathrm{~mm}$. According to JIS R 7601 standard, the mechanical characteristics of the carbon fiber $\mathrm{C} 100 \mathrm{U}$ were: tensile strength $4900 \mathrm{MPa}$, tensile modulus $240 \mathrm{GPa}$ and elongation at break of $2.0 \%$.

Table 1 Box-beam stacking and dimensions

\begin{tabular}{|c|r|r|}
\hline Surface & Lay-up sequence & Dimensions \\
\hline Upper surface & {$[30 / 90 / 30 / 90]_{\mathrm{s}}$} & $220 \times 580 \mathrm{~mm}$ \\
\hline Lower surface & {$[-30 / 90 /-30 / 90]_{\mathrm{s}}$} & $220 \times 580 \mathrm{~mm}$ \\
\hline Leading spar & {$[ \pm 45]_{\mathrm{s}}$} & $50 \times 25 \times 580 \mathrm{~mm}$ \\
\hline Trailing spar & {$[ \pm 45]_{\mathrm{s}}$} & $50 \times 25 \times 580 \mathrm{~mm}$ \\
\hline
\end{tabular}

The epoxy resin used for infusion had a density of $0.98 \mathrm{~g} / \mathrm{cm}^{3}$ and an extremely low viscosity of $500-900 \mathrm{cP}$ at $25^{\circ} \mathrm{C}$. Mixed with the $\mathrm{H} 283$ hardener in a ratio of 18:100, the resin had a working time of 50 minutes.

The upper and the lower panels are the tailored parts of the wing box since they are the primary load carrying pieces. With the mirrored stacking of the two panels (circumferentially asymmetrical stiffness box beam) [21] the bend-twist coupling stiffness coefficient $c_{16}$ will be maximized, thus maximizing also the $\beta$ coefficient. The stacked laminas for the upper and the lower panels are presented in Fig. 2. They were enclosed in a plastic bag to which a vacuum compressor was connected allowing for the resin mixture to flow into the bag and to impregnate the fabric (Fig. 3). The panels were then left for 48 hours in vacuum to cure at ambient temperature.

The spars, being loaded primarily in shear, were fabricated as symmetric $\mathrm{C}$ section profiles with orthotropic behavior so that they don't alter the general bend-twist coupling (Fig. 4).

The resulting panels and spars were trimmed to the prescribed dimensions and assembled using a special mono-component adhesive TEROSON MS9220 (Fig. 5).

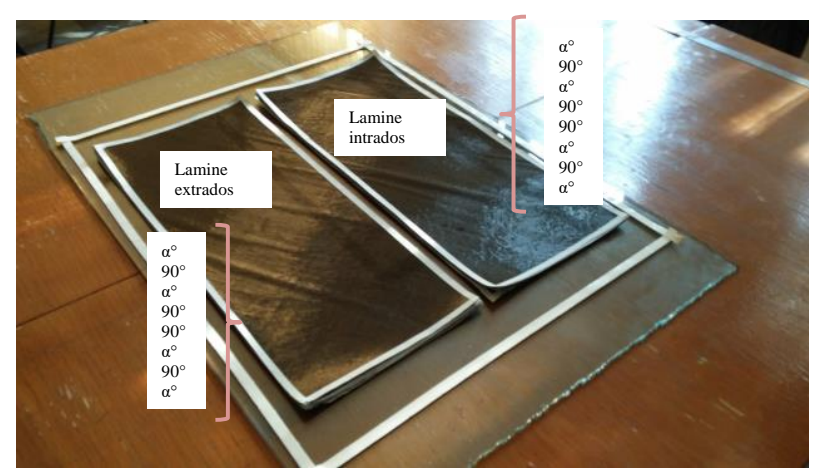

Fig. 2. Upper and lower panel lay-up

For the experimental setup, one end of the wing box was clamped in a mixture of resin and hardener that cured and provided a $25 \mathrm{~mm}$ thick stiff base for the cantilever wing. The thickness of the base was carefully chosen so that an uncontrolled build-up of heat was avoided.

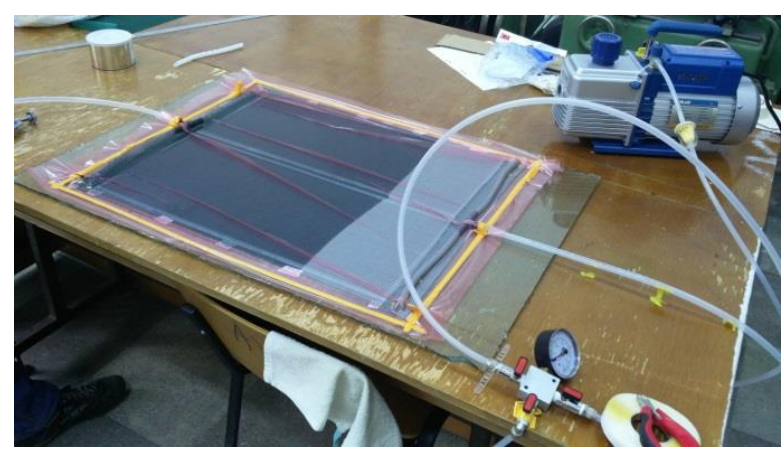

Fig. 3 Lower and upper panels VARTM assembly

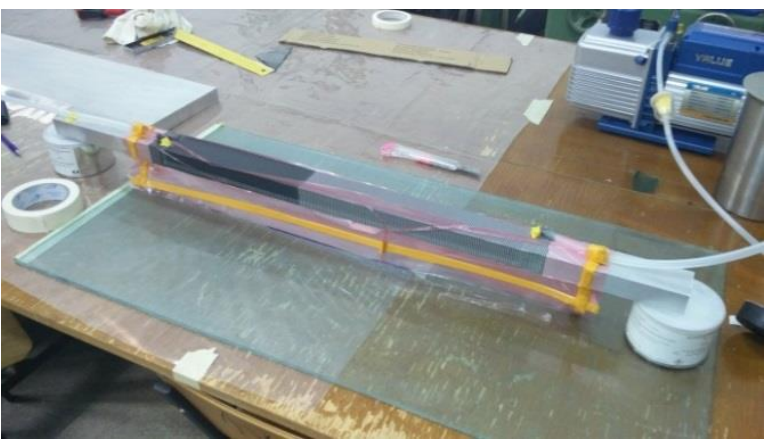

Fig. 4 The $\mathrm{C}$ section spar VARTM assembly

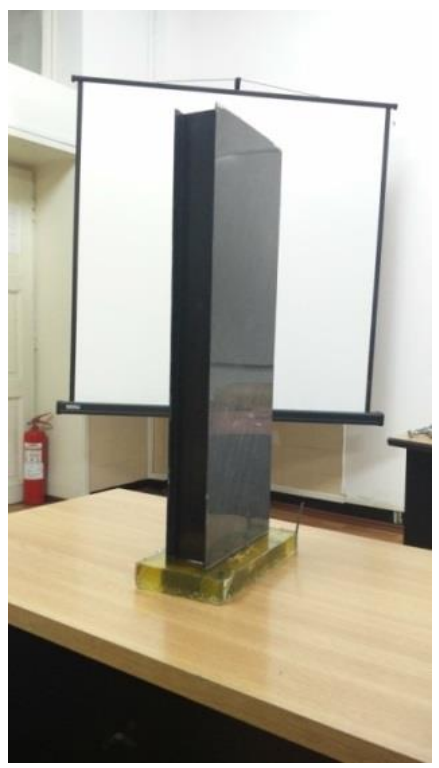

Fig. 5 The composite wing box

\section{Wing Box Testing}

The cantilever wing box has been extensively tested and the experimental data was gathered to create a calibrated finite element model of the wing. The experimental deformed shapes were compared with the analytical solution from the preliminary design tool and with the numerical FEM solution.

A finite element analysis was completed in ANSYS 18.1 using the special composite materials module Composite Prep/Post (ACP). The finite element model of the wing box used SHELL181 elements which has good results 
when used on homogeneous materials with small deformations, very small shell thickness and small warping. One unidirectional lamina was defined in ACP module for the composition of all four parts of the wing box.

The mechanical properties of the unidirectional lamina were determined from laminated plates made of plies stacked at $0^{\circ}$.

To this scope the plates were instrumented with 8 Dytran $3225 \mathrm{M} 24 \mathrm{~T}$ miniature accelerometers connected to a Quantum MX840 acquisition system for an operational modal analysis (OMA). Unlike experimental modal analysis, OMA does not need to know and/or to control the input excitation. Without the need of any vibration shaker or impact hammer, the excitation of the structure is done with a white noise just by tapping randomly in time and also spatially.

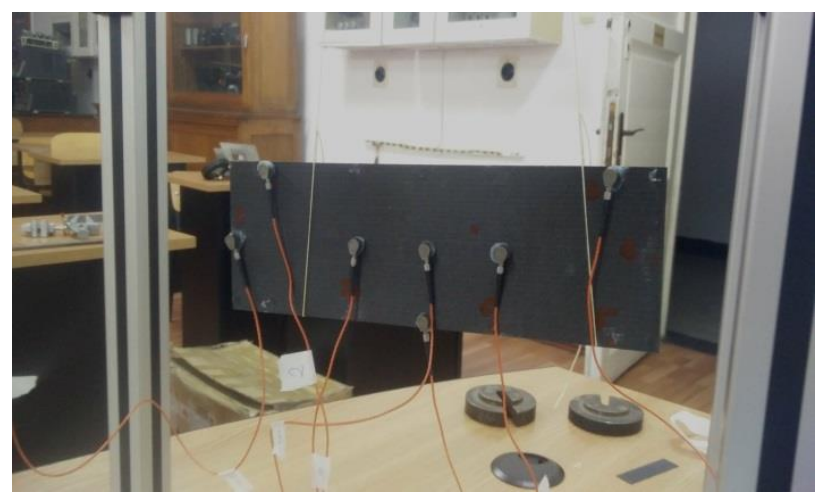

Fig. 6 Instrumentation of the $0^{\circ}$ laminated plate for OMA

The plates were suspended on elastic strings for free-free boundary conditions and the response to random taps was captured by accelerometers. The data was processed with the OMA module of the ARTEMIS software, separating the noise and inputs from the outputs and returning the unbiased modal information only.

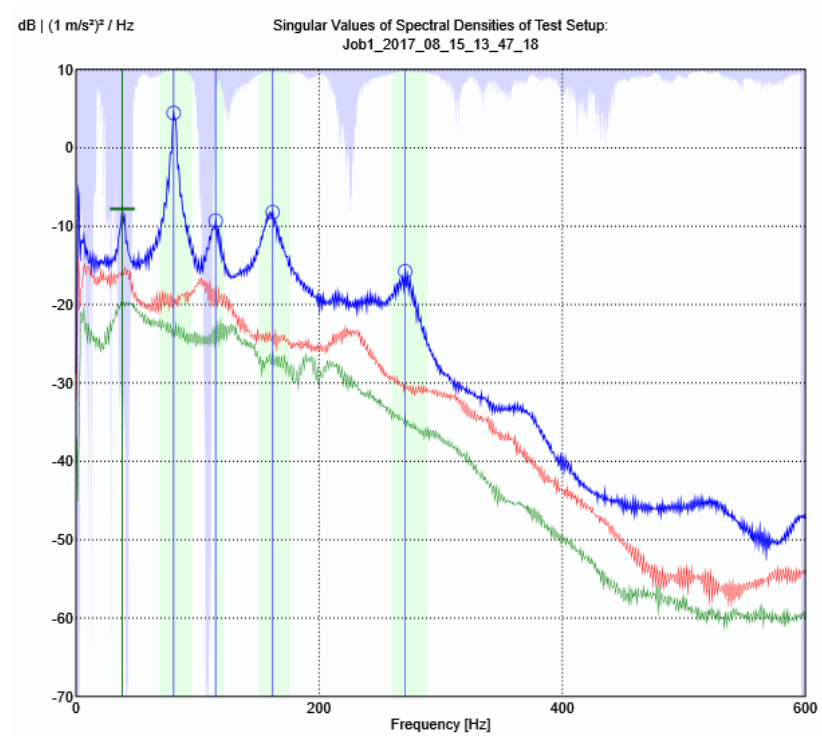

Fig. 7 Natural frequencies of the $0^{\circ}$ laminated plates computed with ARTEMIS OMA
The first four modal shapes extracted are presented in Table 2. We notice that one other advantage of the OMA method is the possibility of computing also the damping of each mode and its complexity.

Table 2 Modal characteristics of the $0^{\circ}$ laminated plates

\begin{tabular}{|c|c|c|}
\hline Frequency [Hz] & Damping [\%] & Complexity [\%] \\
\hline 40.43 & 2.41 & 2.235 \\
\hline 82.03 & 4.64 & 3.265 \\
\hline 118.40 & 4.86 & 7.352 \\
\hline 164.65 & 3.76 & 14.737 \\
\hline
\end{tabular}

The material characteristics can be identified from these responses using the FEMTOOLS software. The four resonance frequencies are enough for the material identification of an orthotropic material like the $0^{\circ}$ laminated plate. Since the structure under test consists of just one material and no local stiffness or mass changes are present, the only unknowns are $E_{11}, E_{22}, G_{12}$ and $v_{12}$ if we derive the density from the geometrical data and the mass of the plates. The procedure consists in generating a mesh for the plate geometry and an initial estimation of the material properties. After a normal modes analysis, the calculated modal shapes are compared with the experimental data based on a parameter and response definition. Assuming a linear behavior, the material properties are updated in an iterative process until the convergence criteria is attained. Table 3 gives the initial estimates of the $0^{\circ}$ laminated plate material characteristics and those obtained with FEMTOOLS. The density was calculated from the known dimensions and mass.

Table 3 Material identification

\begin{tabular}{|c|r|r|r|r|r|}
\hline & \multicolumn{1}{|c|}{$\begin{array}{c}\boldsymbol{\rho} \\
(\mathbf{k g} / \mathbf{m 3})\end{array}$} & $\begin{array}{c}\mathbf{E}_{\mathbf{1 1}} \\
(\mathbf{G P a})\end{array}$ & $\begin{array}{c}\mathbf{E}_{\mathbf{2 2}} \\
(\mathbf{G P a})\end{array}$ & $\begin{array}{c}\mathbf{G}_{\mathbf{1 2}} \\
(\mathbf{G P a})\end{array}$ & $\mathbf{v}_{\mathbf{1 2}}$ \\
\hline $\begin{array}{c}\text { Initial } \\
\text { guess }\end{array}$ & 1290 & 121 & 8.60 & 4.70 & 0.27 \\
\hline $\begin{array}{c}\text { Model } \\
\text { updating }\end{array}$ & 1290 & 82 & 5.83 & 3.01 & 0.27 \\
\hline
\end{tabular}

Once the material characteristics determined, the finite element model of the wing box was further calibrated with FEMTOOLS again. Obviously the fixed boundary condition is an idealization of the real clamped condition which does not have infinite stiffness and also may include some thermal stress due to the cooling of the rigid base made of epoxy.

Using the same accelerometers and acquisition data system, the cantilever wing box was instrumented and the the modal shapes were extracted using OMA and ARTEMIS. For a better representation of the modal shapes, 4 out of the 8 accelerometers were kept fixed for reference and the other 4 were rolled six times in different positions having a total of 28 measured responses. The Modal Assurance Criterion matrix presented in Fig. 8 confirms the orthogonality of the identified modal shapes.

The extracted modes were used to update the finite element model by adjusting the stiffness of the clamped 
nodes until the calculated modal shapes were as close as possible to the experimental modes. Looking at Table 4 we can still notice an error of $4-7 \%$ on the first four resonant frequencies which can be attributed to the adhesive added mass which is not accounted for in the FEM model and also to the stiffness of the bonding.

Table 4 FEM updating. The first 4 modal shapes
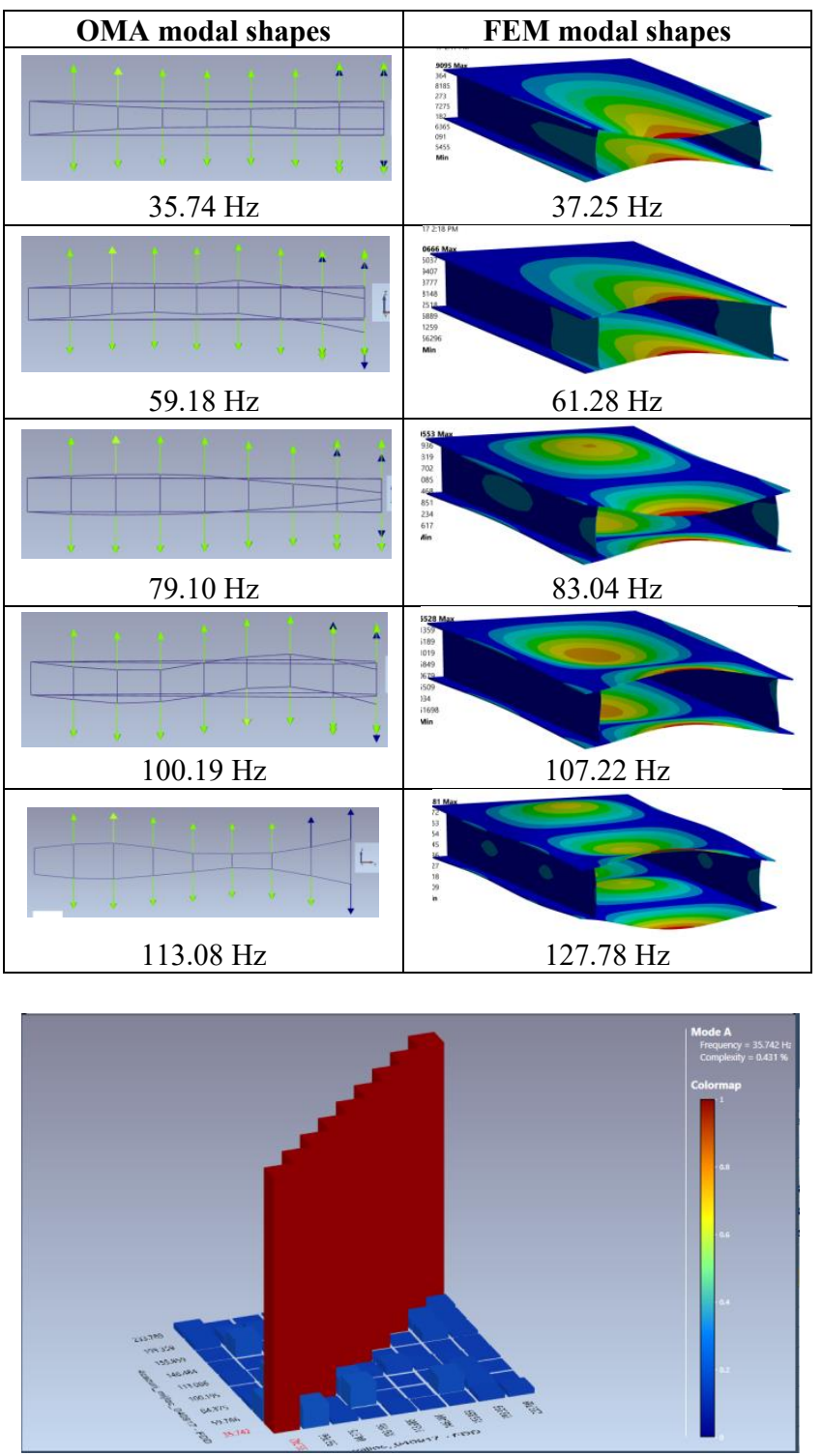

Fig. 8 Modal assurance criterion matrix

The calibrated finite element model was used to simulate the deformation of the composite wing box under simple loads and to compare it with the bend (Fig. 9) and the twist (Fig. 10) predicted by the preliminary design tool.

By definition, the preliminary design tool represents the wing as a beam. To compare data, the FEM used as reference line the symmetry axis of the wing box to extract bending and twist results.

The graphs show that there is good correlation between the numerical and analytical models. The bending response along the span of the wing is very well represented by the analytical model, the error being considered acceptable in terms of preliminary designs usage of analytical tool. When analyzing the twist response one can see that the analytical tool is overestimating the response at the fixed end of the wing but after approximately four characteristic lengths (wing chord) from the clamped end the response is similar. Obviously, the analytical tool will not be able to predict the local response at the fixed end but the overall twist response is in good agreement with the FEM response.

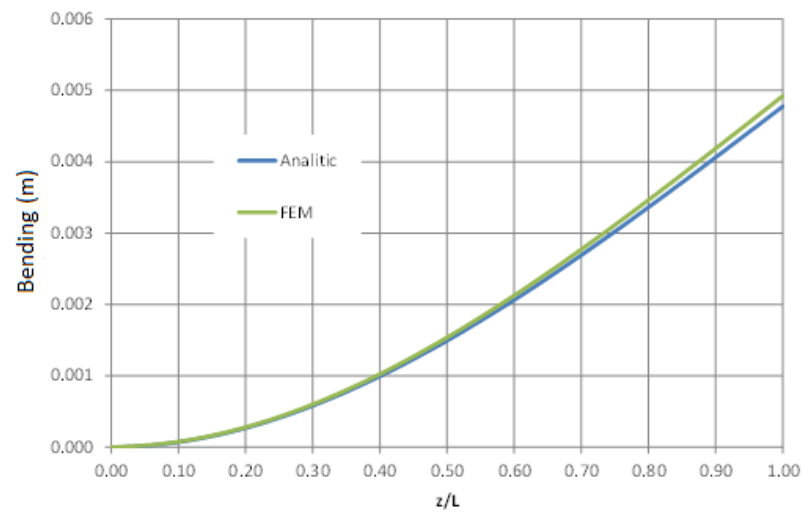

Fig. 9 Bending response - FEM vs. analytical

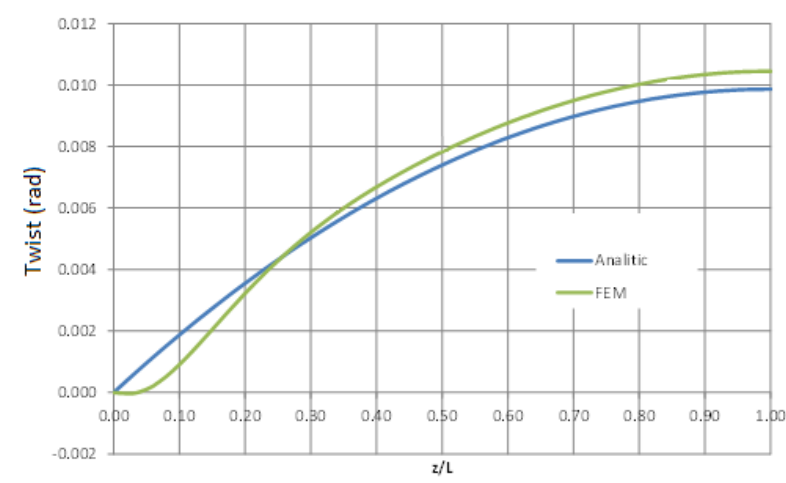

Fig. 10 Twist response - FEM vs. analytical

\section{Conclusions}

A preliminary design tool was developed for the aeroelastic tailoring of composite wings. The computationally intensive task is simplified in the preliminary design phase by the idealization of the wing as a beam with a constant cross-section. The optimization procedure proposed here controls the bendtwist coupling stiffness through the $\beta$ coefficient and determines the bend and the twist under simple aerodynamic loads using as input data the lay-up of the upper and lower surfaces of the wing.

For the validation of the tool, a test model was fabricated and tested. Using a vacuum assisted resin transfer method, a 220x580x50mm wing box was assembled from laminated panels with circumferentially asymmetrical lay-ups. The experimental data was used to calibrate the finite element model of the wing box with the same dimensions and the same stacking in the panels. Two less common methods were used in this regard, one involving the determination of modal frequencies by 
operational modal analysis and the second involving the material characteristics identification by model updating techniques.

The bending and the twist response of the preliminary design tool were in good correlation with the deformation of the calibrated finite element model confirming the validity of the new tool that can be used to quickly design simple composite wings that would exhibit a controllable bend-twist effect. Once the aeroelastic objective is defined - whether reduced drag or increased lift, higher divergence speed of flutter speed, increased maneuverability or increased efficiency of control surfaces - the tool can be used to evaluate optimization variables like laminas properties, stacking order and/or ply angles in order to gain quickly an insight of the aeroelastic response.

The preliminary design tool can be further improved, though not without technical difficulties, to include all the generalized displacements and variable crosssections.

This work was supported by a grant of the Ministry of Innovation and Research, UEFISCDI, project number 4SOL/2017 within PNCDI III.

\section{References}

1. R. Bucci, C. Warren, E. Starke, Need for New Materials in Aging Aircraft Structures, Journal of Aircraft, 37, pp. 122-129 (2000)

2. J. Renton, D. Olcott, B. Roeseler, R. Batzer, B. Baron, A. Velicki, , Future of Flight Vehicle Structures (2002-2023), Journal of Aircraft, 41, pp. 986-998 (2004)

3. C.V. Jutte, B.K. Stanford, Aeroelastic Tailoring of Transport Aircraft Wings: State-of-the-Art and Potential Enabling Technologies, NASA/TM-2014218252 (2014)

4. M. Kameyama, H. Fukunaga, Optimum Design of Composite Wings for Aeroelastic Characteristics using Lamination Parameters, Computers and Structures, 85, pp. 213-224 (2007)

5. D. Kim, S. Oh, I. Lee, J. Kweon, J. Choi, Weight Optimization of Composite Flat and Curved Wings Satisfying Both Flutter and Divergence Constraints, Key Engineering Materials, 334, pp. 477-480 (2007)

6. D. Leon, C. de Souza, J. Fonseca, R. Silva, Aeroelastic Tailoring Using Fiber Orientation and Topology Optimization, Structural and Multidisciplinary Optimization, 46, pp. 663-677 (2012)

7. S. Guo, W. Cheng, D. Cui, Aeroelastic Tailoring of Composite Wing Structures by Laminate Layup Optimization, AIAA Journal, 44, pp. 3146-3149 (2006)

8. S. Guo, Aeroelastic Optimization of an Aerobatic Wing Structure, Aerospace Science and Technology, 11, pp. 396-404 (2007).
9. H. Ghiasi, D. Pasini, L. Lessard, Optimum Stacking Sequence Design of Composite Materials, Part I: Constant Stiffness Design, Composite Structures, 90, pp. 1-11 (2009)

10. A. Attaran, D. Majid, S. Basri, A. Mohd Rafie, E. Abdullah, Structural Optimization of an Aeroelastically Tailored Composite Flat Plate Made of Woven Fiberglass/Epoxy, Aerospace Science and Technology, 15, pp. 393-401 (2011)

11. M. Shirk, T.J. Hertz and T.A. Weisshaar, Aeroelastic tailoring - Theory, practice, and promise, Journal of Aircraft, 23, pp. 6-18 (1986).

12. S. Kuzmina, G. Amiryants, J. Schwseiger, J. Cooper, M. Amprikidis, O. Sensburg, Review and Outlook on Active and Passive Aeroelastic Design Concepts for Future Aircraft, International Council of the Aeronautical Sciences/AIAA (2002)

13. S.N. Jung, ,V.T. Nagaraj, I. Chopra, Refined structural model for thin- and thick-walled composite rotor blades, AIAA Journal , 40, pp. 105116 (2002)

14. L. Cavagna, S. Ricci, L. Riccobene, A Fast Tool for Structural Sizing, Aeroelastic Analysis and Optimization in Aircraft Conceptual Design, AIAA Structures, Structural Dynamics, and Materials Conference (2009)

15. E. Johnson, V. Vasiliev and D. Vasiliev, Anisotropic thin-walled beams with closed crosssectional contours, AIAA Journal, 39, pp. 2389$2393(2001)$

16. E. Ferede, M. Abdalla, Cross-sectional modelling of thin-walled composite beams, 55th AIAA/ASME/ ASCE/ AHS/ ASC Structures, Structural Dynamics, and Materials Conference (2014)

17. V. Vasiliev, E. Morozov, Advanced Mechanics of Composite Materials and Structural Elements, Elsevier, 2013

18. Z. Qin, P. Marzocca, L. Librescu, Aeroelastic Instability and Response of Advanced Aircraft Wings at Subsonic Flight Speeds, Aerospace Science and Technology, 6, pp. 195-208 (2002)

19. L. Rehfield and R. Cheung, "Some Basic Strategies for Aeroelastic Tailoring of Wings with Bend-Twist Coupling: Part One", 44th AIAA/ ASME/ ASCE/ AHS/ ASC Structures, Structural Dynamics, and Materials Conference (2003)

20. M. Mihaila-Andres, P.V. Rosu, C.M. Larco and R.C. Pahonie, Aeroelastic Tailoring of Thin-Walled, Closed-Section Wing Box with Bend-Twist Coupling, 8th International Conference on Mechanical and Aerospace Engineering (ICMAE) pp. 345-349 (2017)

21. H. Ghiasi, K. Fayazbakhsh, D. Pasini, L. Lessard, Optimum Stacking Sequence Design of Composite Materials, Part II: Variable Stiffness Design Composite Structures, 93, pp. 1-13 (2010). 